
15 Hospital. 3JH, UK.

\title{
A rare $P A N K 2$ deletion in the first North African patient affected with pantothenate kinase associated neurodegeneration
}

Stephanie Efthymiou ${ }^{1,2}$, Yamna Kriouile ${ }^{4}$, Vincenzo Salpietro ${ }^{1}$, Rhouda Hajar ${ }^{4}$, Zouiri Ghizlane ${ }^{4}$, Kshitij Mankad ${ }^{3}$, Mohamed El Khorassani ${ }^{4}$, Mhammed Aguennouz ${ }^{5}$, SYNaPS Study Group, Henry Houlden ${ }^{1}$, Sarah Wiethoff ${ }^{1,6}$

${ }^{1}$ Department of Neuromuscular Disorders, ${ }^{2}$ Department of Clinical and Experimental Epilepsy, UCL Institute of Neurology, Queen Square, London WC1N 3BG, UK.

${ }^{3}$ Department of Neuroradiology, Great Ormond Street Hospital for Children, London WC1N

${ }^{4}$ Unit of Neuropediatrics and Neurometabolism, Pediatric Department 2, Rabat Children's

${ }^{5}$ Department of Clinical and Experimental Medicine, University of Messina, Sicily.

${ }^{6}$ Center for Neurology and Hertie Institute for Clinical Brain Research, Eberhard Karls-University, Tübingen, Germany.

\section{Corresponding author:}

Sarah Wiethoff, MD, PhD; s.wiethoff.12@ucl.ac.uk

\section{References: 10}

\section{Word count: 1173}

Keywords: brain iron accumulation; neurodegeneration; PANK2; NBIA; North Africa

\section{Dear Editor,}

Neurodegeneration with brain iron accumulation (NBIA) disorders are a heterogeneous set of inherited, rare and clinically diverse neurological diseases often characterised by neuropathology of the basal ganglia as a consequence of iron deposition. They are usually childhood-onset genetic conditions and the majority of affected individuals present with developmental delay,

4 abnormal behavior, progressive cognitive impairment and pyramidal/extrapyramidal movement 
disruption. Post-mortem pathology highlights axonal swellings with ubiquitinated aggregates, tau

36 tangles or Lewy bodies, depending on the NBIA subtype [1].

37 Variants in at least 10 genes have been established to cause NBIA disorders. Each of these

38 disease genes encode a protein with distinct cellular functions, including regulation of iron

39 metabolism, mitochondrial metabolism, lipid homeostasis and autophagy [2]. The most common

40 NBIA subtype, accounting for $35-50 \%$ of NBIA cases [2, 3] is pantothenate kinase-associated

41 neurodegeneration (PKAN) caused by biallelic variants in PANK2 (MIM \#606157). PANK2 was

42 the first causal gene discovered in NBIA, with cases reported from nearly all continents [1, 4-8].

$43 P A N K 2$ encodes a mitochondrial protein implicated in the synthesis of coenzyme A (CoA), an

44 important molecule for an efficient metabolism of the cell.

45 The clinical entity PKAN can be divided into atypical and typical PKAN. Typical PKAN

46 patients show early childhood-onset, severe presentation and more rapid progression. The

47 mutational spectrum includes homozygous variants causing protein truncation more often than in

48 atypical, later-onset PKAN cases, where variants tend to be compound heterozygous and more

49 often result in amino acid changes [9]. Later disease onset and speech defects as well as

50 psychiatric and cognitive decline are observed more often in atypical cases [10].

51 Here we report a novel PANK2 homozygous deletion in a Moroccan girl with a typical PKAN

52 phenotype. To the best of our knowledge this report represents the first PKAN case from North

53 Africa. The proband, a 10 year old girl, was born from first degree consanguineous parents. History

54 of previous neurological or genetic diseases was unremarkable in the family (Figure 1A). She was

55 born at full-term without birth injury and in good health. At the age of 16 months, she presented

56 with loss of walking and standing, imbalance and frequent forward head falls. She presented with

57 frontal humps and scars during her clinical visit. Examination of the nervous system revealed

58 spasticity and cognitive delay. At 7 years of age, she developed sphincter dysfunction, athetosis of

59 the upper limbs and 4-limb dystonia which later on spread to involve trunk, neck and face with

60 opisthotonus, oromandibular dystonia and severe retrocollis. Her language and speech was normal

61 until the age of 7 after which her speech regressed and became slow with dysarthria, but no

62 stuttering. She also presented with behavioural disturbances including agitation, irritability and

63 significant sleep disorder with frequent awakenings leading to insomnia. The patient received

64 psychomotor rehabilitation and physiotherapy in conjuction with high-dose baclofen administered

65 through oral route. Adjunct pharmacological therapy included trials of haloperidol, 1-dopa and 
benzodiazepines that were largely non-effective. No intrathecal baclofen or DBS was available to

67 her.

68 Laboratory tests including blood biochemistry, ceruloplasmin, thyroid function, parathormone,

69 calcitonin, serum anti-HIV antibodies, anti-syphilis antibodies and autoimmune antibodies were

70 normal. Brain MRI (1.5 Tesla) performed at the age of 9 revealed mild diffuse cortical atrophy as

71 well as symmetric mineralisations of the bilateral globus pallidus and central hyperintense foci

72 termed as 'eye of the tiger' sign. In essence, there is evidence of blooming artefact within the globi

73 pallidi appearing 'dark' on the axial image along with the central gliosis appearing hyperintense

74 or 'bright'. (Figure 1D i, T2 WI). These foci are consistent with regions of vacuolisation and 75 gliosis, as suggested in previously reported pathology literature on PANK2 variants (Figure 1D ii).

76 There was no evidence of cerebellar atrophy, or calaval hypertrophy as described in other variants

77 such as PLA2G6 associated with brain iron accumulation (Figure 1D iii). Lumbar MRI at age 9

78 showed apophyseal joint damage and intervertebral disc degeneration at the L3-L4, L4-L5, L5-L6

79 spinal segments without any associated clinical phenotype.

80 Written informed consent was obtained from the patient and her parents, after which DNA was

81 extracted from peripheral lymphocytes from father and index patient according to a standard

82 protocol of phenol-chloroform extraction. DNA of the mother was unfortunately not available.

83 WES was performed as previously described [11] in both the affected female and the father

84 (Figure 1A: II-1, I-1) as well as a healthy control from our in-house control database. In brief,

85 Nextera Rapid Capture Enrichment kit (Illumina) was used according to the manufacturer

86 instructions. Libraries were sequenced in an Illumina HiSeq3000 platform using a 100-bp paired-

87 end reads protocol. Sequence alignment to the human reference genome (UCSC hg19), and

88 variants call and annotation were performed as described elsewhere [11]. In total, 81,799,534 (II-

89 1) unique reads were generated. All synonymous and in-silico predicted benign changes were

90 discharged. The raw list of single nucleotide variants (SNVs) and indels was then filtered. Only

91 exonic and donor/acceptor splicing variants were considered. In accordance with the pedigree

92 and phenotype, priority was given to rare variants $[<1 \%$ in public databases, including 1000

93 Genomes project, NHLBI Exome Variant Server, Complete Genomics 69, and Exome

94 Aggregation Consortium (ExAC v0.2)] that were fitting a recessive model.

95 The only homozygous variant in a disease-causing gene that we identified was a homozygous

96 frameshift deletion in PANK2 exon 3 (NM_024960.6:c.303_304delAG; NP_001311120.1: 
p.Val103Terfs, dbSNP rsID: rs778550409, ClinVar variant ID: 456524) The two base-pair

98 deletion at nucleotide position 303-304 causes a corresponding frameshift at codon 101 and

99 results in a premature stop codon at codon 103. This deletion in PANK2 emerged as the most

100 likely explanation for the child's phenotype. This deletion has been reported once before

101 according to ClinVar and according to published databases is not frequently implicated in

102 PKAN. It is predicted to be disease-causing on Mutation Taster $(\mathrm{p}=1)$ and deleterious on SIFT

$103(\mathrm{p}=0)[12,13]$. Segregation analysis at the DNA level performed by traditional Sanger sequencing

104 (processed on an ABI 3730 analyser and analysed on Sequencher 4.1.4) confirmed the variant as

105 homozygous in the proband and heterozygous in the father (Figure 1B). For segregation analysis

106 by Sanger sequencing BigDye terminator v3.1 cycle sequencing chemistry (Applied Biosystems,

107 Weiterstadt, Germany) was used with PCR and sequencing primers as follows: Forward (5'-

108 CGGATTCAATGGACGGTCAC -3') and Reverse (5'- CCTAACAGGTTCTTGAAGGTGT 109 3').

110 The current study identified a rare homozygous deletion in PANK2 (c.303_304delAG,

111 p.Val103Terfs) leading to a premature stop codon in a Moroccan patient with a typical NBIA

112 disorder. The deletion of 2 nucleotides at position 303-304 causes a change in the reading frame

113 with premature termination of translation two codons later at codon 103. This is expected to be

114 resulting in an absent or highly disrupted protein suggesting a severe loss of gene function

115 mechanism. Loss-of-function variants in PANK2 are known to be pathogenic. To date, more than

116100 pathogenic $P A N K 2$ variants have been described in PKAN patients around the globe but, to

117 the best of our knowledge, the disease has not been described in the North-African population so

118 far. This study further expands the PANK2 ethnic and clinical spectrum and reports the first

119 PKAN case associated with c.303_304del variant in Morocco. This information can help with

120 the genetic screening of north African patients presenting typical PKAN features which could

121 lead to more accurate genetic diagnoses and help in genetic counseling as well as, potentially in

122 the future, prenatal diagnoses in the suspected families

123

124 Conflict of interest

125 The authors declare no conflict of interest.

127 Acknowledgements 
128 We gratefully acknowledge our collaborators in Morocco for their enthusiasm in the study.

129 This study was supported in part by The Wellcome Trust in equipment and strategic award

130 (Synaptopathies) funding (WT093205 MA and WT104033AIA). SW is supported by the Ministry 131 of Science, Research and the Arts of Baden-Württemberg and the European Social Fund (ESF) of 132 Baden-Württemberg (31-7635 41/67/1).

133

A

।

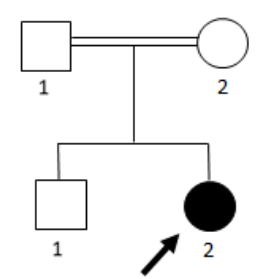

C

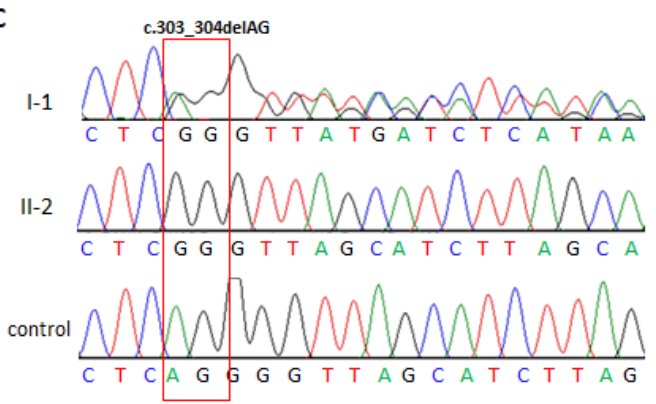

Human Mutant Mouse Rat

Bovin

Horse

Zebrafish
KLPFDLKNPYPLLLVNIGSGVSILAVYSKDNYKRVTGTSLGGGTFFGLCCLLTGCTTFEE KLPFDLKNPYPLLLVNIGS $*$ * KLPFDLKNPYPLLLVNIGSGVSILAVYSKDNYKRVTGTSLGGGTFFGLCCLLTGCSTFEE KLPFDLKNPYPLLLVNIGSGVSILAVYSKDNYKRVTGTSLGGGTFFGLCCLLTGCSTFEE KLPFDLKNPYPLLLVNIGSGVSILAVYSKDNYKRVTGTSLGGGTFFGLCCLLTGCTTFEE KLPFDLKNPYPLLLVNIGSGVSILAVYSKDNYKRVTGTSLGGGTFFGLCCLLTGCTTFEE QKAYNLENPYPLLLVNIGSGVSILAVYSKDNYKRVTGTSLGGGTFLGLCCLLTGCSTFEE

\section{D}

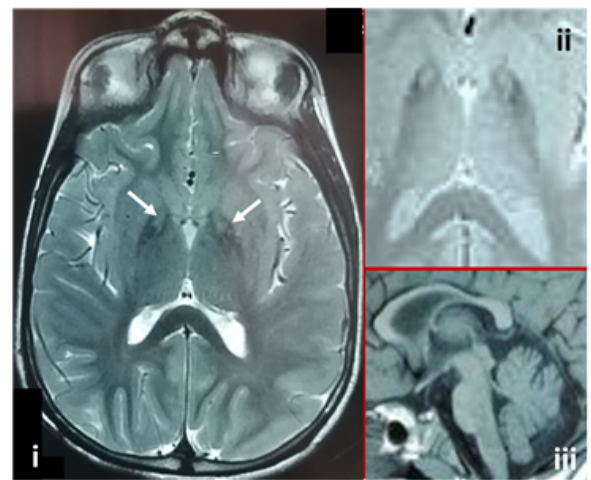

134

Figure 1 (A) Family pedigree (B) Interspecies alignment performed with Clustal Omega shows the complete conservation down to invertebrates of the amino acid residues affected by the deletion. (C) Individual results of Sanger sequencing indicating the proband (II-1) to carry the homozygous PANK2 truncating variant (c.303_304delAG:p.Val103Terfs) while the father (I-1) carries the heterozygous variant. For clarity, only I-1 is shown here, as well as a healthy control homozygous for the reference allele (third lane, wildtype) (D) Axial T2 WI (i), and zoomed-in axial T2* WI (ii) sequences showing hypointense signal return from both globi pallidi, consistent with increased iron deposition. Further, within these areas of hypointensity are defined foci of increased (hyperintense) signal pointed with arrows. These foci are consistent with regions of vacuolisation and gliosis, as suggested in previously reported pathology literature on PANK2 variants. No other areas of increased brain iron accumulation were noted. Note also that there is no evidence of cerebellar atrophy, or calaval hypertrophy on the sagittal T1 WI (iii) as described in other variants (e.g. PLA2GO) associated with brain iron accumulation. 
149 [1] Y.F. Li, H.F. Li, Y.B. Zhang, J.M. Wu, Novel homozygous PANK2 mutation identified in a consanguineous Chinese pedigree with pantothenate kinase-associated neurodegeneration, Biomedical reports 5(2) (2016) 217-220.

152 [2] A. Gregory, S.J. Hayflick, PANK2 mutation screening recommended to confirm diagnosis of pantothenate kinase-associated neurodegeneration, AJNR. American journal of neuroradiology 27(5) (2006) 951.

[3] P. Hogarth, Neurodegeneration with brain iron accumulation: diagnosis and management, Journal of movement disorders 8(1) (2015) 1-13. Pandita, S. Sharma, A variation in PANK2 gene is causing Pantothenate kinase-associated Neurodegeneration in a family from Jammu and Kashmir - India, Scientific reports 7(1) (2017) 4834. genes in patients with neurodegenerative disorders: two case reports, BMC medical genetics $18(1)$

162 (2017) 87.

163 [6] G.P. Paraskevas, C. Yapijakis, A. Bougea, V. Constantinides, M. Bourbouli, E. Stamboulis, E. Kapaki, 164 Novel PANK2 mutation in the first Greek compound heterozygote patient with pantothenate-kinaseassociated neurodegeneration, SAGE open medical case reports 5 (2017) $2050313 \times 17720101$. [7] Y. Zhang, D. Zhou, T. Yang, Novel PANK2 mutation in a Chinese boy with PANK2-associated neurodegeneration: A case report and review of Chinese cases, Medicine 98(4) (2019) e14122. clinical, and radiographic delineation of Hallervorden-Spatz syndrome, The New England journal of medicine 348(1) (2003) 33-40. associated neurodegeneration (PLAN): review of two major neurodegeneration with brain iron accumulation (NBIA) phenotypes, Int Rev Neurobiol 110 (2013) 49-71. [10] S.J. Hayflick, Neurodegeneration with brain iron accumulation: from genes to pathogenesis, 175 Seminars in pediatric neurology 13(3) (2006) 182-5. [11] N.E. Mencacci, E.J. Kamsteeg, K. Nakashima, L. R'Bibo, D.S. Lynch, B. Balint, M.A. Willemsen, M.E. Adams, S. Wiethoff, K. Suzuki, C.H. Davies, J. Ng, E. Meyer, L. Veneziano, P. Giunti, D. Hughes, F.L. Raymond, M. Carecchio, G. Zorzi, N. Nardocci, C. Barzaghi, B. Garavaglia, V. Salpietro, J. Hardy, A.M.

180 PDE10A Cause Childhood-Onset Chorea with Bilateral Striatal Lesions, American journal of human 181 genetics 98(4) (2016) 763-71.

182 [12] R. Vaser, S. Adusumalli, S.N. Leng, M. Sikic, P.C. Ng, SIFT missense predictions for genomes, Nat 183 Protoc 11(1) (2016) 1-9.

184 [13] J.M. Schwarz, D.N. Cooper, M. Schuelke, D. Seelow, MutationTaster2: mutation prediction for the 185 deep-sequencing age, Nature methods 11(4) (2014) 361-2. 